\title{
On a 14-dimensional lattice invariant under the simple group $G_{2}(3)$
}

\author{
Robert A. Wilson \\ School of Mathematical Sciences, \\ Queen Mary, University of London, \\ Mile End Road, London E1 4NS, U.K. \\ email: R.A.Wilson at qmul.ac.uk
}

First draft 19/05/10; this version 07/01/12

\begin{abstract}
We study the 14-dimensional real representation of the finite simple group $G_{2}(3)$ and relate it to the smallest Ree group and representations in characteristic 3 . In particular, we give a set of generators which leads to a new and easy proof that the group is indeed $G_{2}(3)$.
\end{abstract}

\section{Introduction}

The 14-dimensional real representation of the simple group $G_{2}(3)$ is a small example of a phenomenon studied in depth by Kostrkin and Tiep [3], whereby the compact real form of a simple Lie algebra gives rise to an interesting lattice, whose automorphism group is larger than one has a right to expect. This example was worked out in detail by Kostrikin, Kostrikin and Ufnarovskii [2], who began with the group $2^{3 \cdot} \cdot \mathrm{PSL}_{3}(2)$, preserving the compact real form of the Lie algebra of type $G_{2}$. After classifying all the lattices preserved by this group, they used a computer to find an automorphism of order 13 of one of the lattices, which they then used to show that the full automorphism group of the lattice is $2 \times G_{2}(3)$.

In [3, Chapter 8] some variants of this approach are given. One approach uses detailed knowledge of the group $G_{2}(3)$ (including information from the character table and the maximal subgroups) to observe that $G_{2}(3)$ must preserve one of the given lattices. Another constructs explicitly by hand a lattice automorphism of order 3 outside the group $2^{3 \cdot} \mathrm{PSL}_{3}(2)$. In either case, intrinsic properties of the lattice are used to show that the automorphism group is no bigger than $2 \times G_{2}(3)$.

It is the aim of this note to provide some simplifications to these proofs, all of which are quite long and involved. The key observation is that $G_{2}(3)$ is generated 
by two subgroups, of shapes $2^{3 \cdot} \mathrm{PSL}_{3}(2)$ and ${ }^{2} G_{2}(3) \cong \mathrm{PSL}_{2}(8): 3$, intersecting in $2^{3}: 7: 3$. Explicit generators for the former, acting on the compact real form of the Lie agebra of type $G_{2}$, in 14 real dimensions, are given in $[2,3]$ and also in [6]. Explicit generators for the latter, in 7 dimensions over $\mathbb{F}_{3}$, are given in $[4,5]$, and in 7 real dimensions in [7, Section 4.5.4]. Now it turns out that, with small modifications, these two constructions can be combined to give a construction of the 14-dimensional real representation of $G_{2}(3)$.

The proof that the group we construct is indeed isomorphic to $G_{2}(3)$ proceeds by constructing an invariant lattice spanned by an orbit of $2 \times 378$ vectors $\pm v$, so that the group is finite, and then finding a map onto the standard copy of $G_{2}(3)$ as automorphisms of the octonion algebra, such that the kernel is trivial.

It is worth noting that this lattice is also described in the Atlas [1, p. 60], with respect to a completely different basis, so that a subgroup $\operatorname{PSL}_{3}(3)$ fixes one coordinate, and acts monomially on the other 13 .

\section{The action of $2^{3} \cdot \mathrm{PSL}_{3}(2)$ on the Lie algebra}

We begin by summarising the results of [6]. There the compact real form of the Lie algebra of type $G_{2}$ was constructed in such a way that an irreducible subgroup $2^{3} \cdot \mathrm{PSL}_{3}(2)$ of the automorphism group was visible. Indeed, this is really an integral form of the Lie algebra whose automorphism group is exactly $2^{3 \cdot} \cdot \mathrm{PSL}_{3}(2)$.

The underlying real vector space is written as a direct sum of seven 2-spaces, labelled by the elements $t \in \mathbb{F}_{7}$, each spanned by three vectors $u_{t}, v_{t}, w_{t}$ such that $u_{t}+v_{t}+w_{t}=0$. First, let us change the notation by replacing $u, v, w$ by the complex numbers $1, \omega, \bar{\omega}$, where $\omega$ is a primitive cube root of unity, so that the real 2-spaces are identified with complex 1-spaces, and the 14-dimensional real vector space is written as a 7 -dimensional complex space. It is straightforward to check that the following elements $\alpha, \beta, \gamma, \delta$ generate a group $2^{3} \cdot \mathrm{PSL}_{3}(2)$.

$$
\begin{aligned}
\alpha & : \quad x_{t} \mapsto \pm x_{t}, \text { minus sign just if } t \in\{0,3,5,6\} \\
\beta & : \quad x_{t} \mapsto x_{t+1} \\
\gamma: & x_{t} \mapsto \omega x_{2 t} \\
\delta: & x_{1} \mapsto-x_{1}, x_{2} \mapsto-\bar{x}_{2}, x_{4} \mapsto \bar{\omega} \bar{x}_{4}, \\
& \quad x_{0} \leftrightarrow \bar{x}_{3}, x_{5} \leftrightarrow \omega x_{6} .
\end{aligned}
$$

The main result of [6] is that there is a unique (up to real scalar multiplication) 14-dimensional Lie algebra invariant under this group. The product is given by $\left[1_{1}, \omega_{2}\right]=\bar{\omega}_{4}$, and images under the group. Notice that the argument given in [6] actually works in every characteristic except 3. In characteristic 3 there is exactly one other solution (in the notation of [6], we can have $\lambda=\mu=\frac{1}{2}$ ), in which $\left[1_{1}, \omega_{2}\right]=\omega_{4}$. 


\section{Representations of the Ree group ${ }^{2} G_{2}(3)$}

In [4] the subgroup $2^{3}: 7: 3$ was used to construct the Ree groups ${ }^{2} G_{2}\left(3^{2 n+1}\right)$ in their natural 7-dimensional representations in characteristic 3. Now in the case $n=0$, this representation actually lifts to characteristic 0 . An explicit proof of this is given for example in $\left[7\right.$, p. 139], where the well-known isomorphism ${ }^{2} G_{2}(3) \cong$ $\mathrm{PSL}_{2}(8): 3$ is also proved. In fact there are three 7 -dimensional representations of this group, obtained from each other by tensoring with a linear character of the quotient of order 3 .

These representations are given by generators $\alpha, \beta, \gamma, \varepsilon$ of the group acting as follows:

$$
\begin{aligned}
\alpha & : \quad x_{t} \mapsto-x_{t} \text { if } t \in\{0,3,5,6\} \\
\beta & : \quad x_{t} \mapsto x_{t+1} \\
\gamma: & x_{t} \mapsto \lambda x_{2 t} \\
\varepsilon \quad: & x_{t} \mapsto-\frac{1}{2}\left(-x_{-t}+x_{1-t}+x_{2-t}+x_{4-t}\right)
\end{aligned}
$$

where $\lambda=1, \omega, \bar{\omega}$ in the three cases. Reducing these matrices modulo $\theta=\omega-\bar{\omega}$ (so that $-\frac{1}{2}=1$ and $\lambda=1$ ) gives the matrices over $\mathbb{F}_{3}$ as in [4].

In the sequel, we shall not use the facts quoted without proof in this section. We merely use them as motivation for the definition of $\varepsilon$.

\section{An orbit of $2 \times 378$ vectors}

If we now put $\lambda=\omega$ so that the two definitions of $\gamma$ agree, then all the maps $\alpha, \beta, \gamma, \delta, \varepsilon$ act semilinearly on the 7-dimensional complex space. Let $G$ be the group generated by $\alpha, \beta, \gamma, \delta, \varepsilon$. We shall show that $G \cong G_{2}(3)$. This is perhaps not completely obvious from what we have done already, but can be proved easily once we have constructed the lattice.

We show first that the vector $(2 \bar{\omega}, 2,0,0,0,0,0)$ lies in an orbit of just $2 \times$ 378 vectors under the action of $G$. To see this we first calculate the image $(\omega \theta, \omega,-\bar{\omega},-1,-\bar{\omega}, 0,1)$ of this vector under $\varepsilon$, where $\theta=\omega-\bar{\omega}=\sqrt{-3}$, and then use elements of the monomial group $\langle\alpha, \beta, \gamma, \delta\rangle \cong 2^{3} \cdot \mathrm{PSL}_{3}(2)$ to map this to $(0,-\theta, \omega, \omega, \bar{\omega}, 1, \bar{\omega})$. Now we observe that the orbit contains at least

- the $2 \times 42$ images under the monomial group of $(2 \bar{\omega}, 2,0,0,0,0,0)$, and

- the $2 \times 336$ images under the monomial group of $\pm(0,-\theta, \omega, \omega, \bar{\omega}, 1, \bar{\omega})$.

Conversely, we must show that this set of $2 \times 378$ vectors is invariant under $\varepsilon$. It is easy to check that $\varepsilon$ has order 2 , and normalizes the subgroup $\langle\beta, \gamma\rangle \cong 7: 3$, extending it to a group 7:6. Therefore we only need to calculate the images under 
$\varepsilon$ of representatives of each of the 18 orbits of $\langle\beta, \gamma\rangle$ on the vectors (up to sign). Indeed, since $\varepsilon$ has order 2 , the calculation reduces to the following 12 cases.

$$
\begin{aligned}
(2 \bar{\omega}, 2,0,0,0,0,0) & \leftrightarrow(\omega \theta, \omega,-\bar{\omega},-1,-\bar{\omega}, 0,1) \\
(2 \bar{\omega},-2,0,0,0,0,0) & \leftrightarrow(-\omega,-\omega \theta,-\bar{\omega}, 1,-\bar{\omega}, 0,-1) \\
(0,1,-\omega \theta, \omega, \bar{\omega}, 1, \bar{\omega}) & \leftrightarrow(1,-\bar{\omega}, 0,1,-\omega, \omega \theta,-\bar{\omega}) \\
(0,-1, \omega \theta,-\omega, \bar{\omega}, 1,-\bar{\omega}) & \leftrightarrow(\omega \theta, \bar{\omega},-1,-\bar{\omega}, 0,1,-\omega) \\
(0,-1,-\omega \theta, \omega,-\bar{\omega}, 1,-\bar{\omega}) & \leftrightarrow(-\bar{\omega},-1,-\bar{\omega}, 0,1, \omega,-\omega \theta) \\
(0,1,-\omega \theta,-\omega, \bar{\omega},-1,-\bar{\omega}) & \leftrightarrow(1,-\omega,-\omega \theta,-\bar{\omega},-1, \bar{\omega}, 0) \\
(0,-1,-\omega \theta,-\omega,-\bar{\omega},-1, \bar{\omega}) & \mapsto(-\bar{\omega}, 0,1, \omega \theta, \omega, \bar{\omega}, 1) \\
(0,1, \omega \theta,-\omega,-\bar{\omega}, 1, \bar{\omega}) & \mapsto(0,1, \omega \theta,-\omega,-\bar{\omega}, 1, \bar{\omega}) \\
(0,-1, \omega \theta, \omega, \bar{\omega},-1, \bar{\omega}) & \mapsto(\omega \theta, \omega, \bar{\omega},-1, \bar{\omega}, 0,-1) \\
(0,1, \omega, \omega \theta,-\bar{\omega}, 1, \bar{\omega}) & \mapsto(-\bar{\omega}, 0,-1,-\omega,-\omega \theta, \bar{\omega},-1) \\
(0,1,-\omega,-\omega \theta, \bar{\omega}, 1, \bar{\omega}) & \mapsto(-\omega,-\omega \theta, \bar{\omega}, 1, \bar{\omega}, 0,1) \\
(0,1, \omega,-\omega \theta, \bar{\omega}, 1,-\bar{\omega}) & \mapsto(0,1, \omega,-\omega \theta, \bar{\omega}, 1,-\bar{\omega})
\end{aligned}
$$

\section{Reduction modulo $\theta$}

Let $\Lambda$ be the $\mathbb{Z}$-lattice spanned by the $2 \times 378$ vectors listed above (but not their multiples by $\omega$ or $\bar{\omega})$. First note that $\theta \Lambda \subseteq \Lambda$, since by transitivity we need only check one vector, and we have

$$
\begin{aligned}
& \theta(2 \bar{\omega}, 2,0,0,0,0,0)=\quad(2 \bar{\omega}, 2,0,0,0,0,0)+ \\
& \quad(2,0,0,0,0,0,2 \bar{\omega})+(2,0,0,0,0,0,-2 \bar{\omega})+ \\
&(0,2 \omega, 0,0,0,2 \bar{\omega}, 0)+(0,2 \omega, 0,0,0,-2 \bar{\omega}, 0) \in \Lambda
\end{aligned}
$$

The semilinear maps $\alpha, \beta, \gamma, \delta, \varepsilon$ all preserve $\Lambda$ and $\theta \Lambda$, so induce linear maps on $\Lambda / \theta \Lambda$. (The only case in which this is not completely obvious is the element $\delta$ : in this case, multiplying the coordinates by $\theta$ has the effect of changing the sign in the definition of $\delta$ for all those coordinates which involve complex conjugation, namely coordinates $0,2,3,4$; since this sign-change is effected by $\alpha^{\beta^{4}}$, the result follows.)

Now $(4,0,0,0,0,0,0) \in \Lambda$, so $(4 \theta, 0,0,0,0,0,0) \in \theta \Lambda$, and similarly for all seven coordinates. But multiplication by 4 induces the identity map on $\Lambda / \theta \Lambda$, so we may obtain coordinates for $\Lambda / \theta \Lambda$ by reducing the coordinates for $\Lambda$ modulo $\theta$.

We obtain in this way $2 \times 378$ vectors in $\Lambda / \theta \Lambda \cong \mathbb{F}_{3}^{7}$. These fall into two orbits under the monomial group $2^{3} \cdot \mathrm{PSL}_{3}(2)$, as follows.

- $2 \times 42$ vectors of shape $( \pm 1, \pm 1,0,0,0,0,0)$, and

- $2 \times 336$ vectors of shape $(0,0, \pm 1, \pm 1, \pm 1, \pm 1, \pm 1)$. 
Moreover, the natural Hermitian form on $\Lambda$, suitably scaled, induces the natural quadratic form on $\Lambda / \theta \Lambda$, that is the form given by the sum of the squares of the coordinates. Our $2 \times 378$ vectors are then exactly the vectors of norm 2 with respect to this quadratic form.

In this way, we obtain an action of $G$ on a 7 -dimensional orthogonal space over $\mathbb{F}_{3}$. Since the $2 \times 378$ given vectors in $\Lambda$ span $\Lambda$, which is an irreducible lattice, the kernel of the permutation action of $G$ on these $2 \times 378$ vectors is trivial. Since these vectors remain distinct on reduction modulo $\theta$, the kernel of the action of $G$ on $\Lambda / \theta \Lambda$ is also trivial.

\section{Preserving the octonion algebra}

Finally, we observe that the induced actions of $\alpha, \beta, \gamma, \delta, \varepsilon$ are exactly the generators for $G_{2}(3)$ given in [7]: indeed, $\alpha, \beta, \gamma, \delta^{\alpha^{3}}$ are given on page 120 , while $\varepsilon$ is in (4.56) on page 139. For the sake of completeness we include the proof here.

First we define the octonion algebra over $\mathbb{F}_{3}$ by identifying the 7 coordinates of $\Lambda / \theta \Lambda$ with square roots of -1 , labelled $i_{t}$ for $t \in \mathbb{F}_{7}$, and defining the other products by $i_{0} i_{1}=-i_{1} i_{0}=i_{3}$, and images under the action of $\beta$ and $\gamma$. Then the defining products are all of the form $i_{r} i_{s}=i_{t}$, where $\{r, s, t\}$ is one of the 7 triples $\{x, x+1, x+3\}$. Since these are the lines which define the Fano plane, and $\alpha$ negates the coordinates corresponding to the complement of a line, we see that $\alpha$ negates exactly two of the three terms $i_{r}, i_{s}, i_{t}$, so preserves the octonion product.

Checking that $\delta$ preserves the product is a little more tedious, but since the permutation part is an automorphism of the Fano plane, it is ony the signs which need checking. Finally, $\varepsilon$ normalizes the group generated by $\beta$ and $\gamma$, so it suffices to check that $\varepsilon$ maps the equation $i_{0} i_{1}=i_{3}$ to a true statement. In other words we need to check that

$$
\left(-i_{0}+i_{1}+i_{2}+i_{4}\right) \cdot\left(-i_{6}+i_{0}+i_{1}+i_{3}\right)=-i_{4}+i_{5}+i_{6}+i_{1} .
$$

This shows that our five generators all preserve the octonion algebra, so lie in $G_{2}(3)$. Since $2^{3} \cdot \mathrm{PSL}_{3}(2)$ is maximal in $G_{2}(3)$, they generate $G_{2}(3)$. (This can also be shown by explicit computations.) This completes the proof that the given matrices in real 14-space do indeed generate a group isomorphic to $G_{2}(3)$.

\section{The minimal vectors of the lattice}

We have not however proved that the full automorphism group of the lattice $\Lambda$ is $2 \times G_{2}(3)$, since it is not obvious that the full automorphism group preserves $\theta \Lambda$, let alone the octonion product on $\Lambda / \theta \Lambda$. The method used in $[2,3]$ is to 
show that the minimal vectors of $\Lambda$ are precisely the $2 \times 378$ vectors listed above, although the proof of this is omitted in [3].

Our 'complex' notation permits a relatively straightforward proof of the salient facts.

Lemma 1 If $v, w \in \Lambda$, then $\Re(v . w) \in 2 \mathbb{Z}$, where $v$.w denotes the usual Hermitian inner product.

Proof. By transitivity it is sufficient to check the inner products of $(2 \bar{\omega}, \pm 2,0,0,0,0,0)$ with the spanning vectors. The result is clear for all vectors in the 84-orbit of the monomial group, so it is sufficient to check all rotations of $(0,-\theta, \omega, \omega, \bar{\omega}, 1, \bar{\omega})$, $(0,1,-\omega \theta, \omega, \bar{\omega}, 1, \bar{\omega}),(0,1, \omega, \omega,-\bar{\omega} \theta, 1, \bar{\omega})$. But the difference of any two of these three vectors is in the 84-orbit, so we only need to check rotations of one of them. This is an easy exercise.

As an immediate corollary, we have that the norm of every vector in $\Lambda$ is a multiple of 4 .

Lemma 2 There is no vector of norm 4 in $\Lambda$.

Proof. Suppose that $v \in \Lambda$ has norm 4. If $v$ has a single non-zero coordinate, then we can find a vector of shape $\left(2^{2}, 0^{5}\right)$ in $\Lambda$, whose inner product with $v$ is $2 \omega$, which is a contradiction. Otherwise, $v$ has at least three zero coordinates, and we may assume two of these are consecutive, so that $v=(x, 0,0, \ldots)$ with $x \notin 2 \mathbb{Z}[\omega]$. Then taking inner products with $(2 \bar{\omega}, 2,0,0,0,0,0)$ and $(2,0,2 \omega, 0,0,0,0)$ yields that both $\Re(x)$ and $\Re(\bar{\omega} x)$ are integers. This is a contradiction.

Lemma 3 There are exactly 756 vectors of norm 8 in $\Lambda$.

Proof. The same argument shows that if $v$ is any vector of norm 8 which has at least two zero coordinates, then the coordinates immediately before and after a string of zeroes are both divisible by 2 . Therefore the only possible distributions of the norm among the coordinates are $\left(4^{2}, 0^{5}\right)$ or $\left(3,1^{5}, 0\right)$.

In the first case, we may assume $v=2(a, b, 0,0,0,0,0)$, where $a, b$ are units in $\mathbb{Z}[\omega]$. Taking inner products with $(0,1,-\omega \theta, \omega, \bar{\omega}, 1, \bar{\omega})$ and $(\bar{\omega}, 0,1,-\omega \theta, \omega, \bar{\omega}, 1)$, shows that $b= \pm 1$ and $a= \pm \bar{\omega}$.

In the second case, a similar argument taking inner products with vectors of shape $\left(2^{2}, 0^{5}\right)$ shows that the powers of $\omega$ in the coordinates of $v$ are the same as in the known spanning vectors of $\Lambda$. Now if we could change sign in one coordinate, we would have a vector of norm 4 in $\Lambda$, so this does not happen. Moreover, changing sign in two coordinates can only happen if the powers of $\omega$ agree with those in one of the vectors of the 84-orbit. This restriction means that the group of allowable sign-changes to $v$ has order at most 16 . But we already have 16 sign combinations for the listed spanning vectors of this type. Thus there can be no more. 


\section{The automorphism group of the lattice}

Now to complete the proof that the automorphism group of the lattice is no bigger than $2 \times G_{2}(3)$, we first compute the real parts of the inner products of a selection of minimal vectors, and find some values $\pm 8, \pm 4, \pm 2$, and 0 for this quantity. Hence the rank of the action of the automorphism group on the 378 pairs of opposite vectors is at least 4 . (In fact the distribution of these values for one minimal vector with all the minimal vectors is $-8^{1}-4^{52}-2^{208} 0^{234} 2^{208} 4^{52} 8^{1}$, but we shall not need this.)

We have already shown that Aut $\Lambda$ has a subgroup $G_{2}(3)$, acting on an orthogonal 7 -space over $\mathbb{F}_{3}$ in such a way that the minimal vectors of the lattice correspond to vectors of norm 2 in this orthogonal space. Moreover, it is known, or can be readily calculated, that the suborbits of $G_{2}(3)$ on the 378 pairs of vectors of norm 2 are $1+52+117+208$. Therefore Aut $\Lambda$ also has suborbits of these sizes. Since the suborbit of size 117 consists exactly of those vectors which are perpendicular to the fixed vector, the projective geometry for the orthogonal group $\mathrm{PO}_{7}(3)$ can be re-constructed from the suborbits. This puts Aut $(\Lambda)$ inside the automorphism group of the orthogonal group, and maximality of $G_{2}(3)$ in $\mathrm{P} \Omega_{7}(3)$ finishes the argument.

\section{References}

[1] J. H. Conway, R. T. Curtis, S. P. Norton, R. A. Parker and R. A. Wilson, An Atlas of Finite Groups, Oxford University Press, 1985.

[2] A. I. Kostrikin, I. A. Kostrikin and V. A. Ufnarovskii, Invariant lattices of type $G_{2}$ and their automorphism groups, Trudy Mat. Inst. Steklov 165 (1984), 79-97; English translation Proc. Steklov Inst. Math. 1985, issue 3(165), 85106.

[3] A. I. Kostrikin and P. H. Tiep, Orthogonal decompositions and integral lattices, de Gruyter, 1994.

[4] R. A. Wilson, An elementary construction of the Ree groups of type ${ }^{2} G_{2}$, Proc. Edinb. Math. Soc. 53 (2010), 531-542.

[5] R. A. Wilson, Another new approach to the small Ree groups, Arch. Math. (Basel) 94 (2010), 501-510.

[6] R. A. Wilson, On the compact real form of the Lie algebra $\mathfrak{g}_{2}$, Math. Proc. Cambridge Philos. Soc. 148 (2010), 87-91.

[7] R. A. Wilson, The finite simple groups, Springer GTM 251, 2009. 